\title{
Beyond the iron group: Heavy metals in hot subdwarfs ${ }^{\star}$
}

\author{
S. J. O'Toole \\ Dr Remeis-Sternwarte, Astronomisches Institut der Universität Erlangen-Nürnberg, Sternwartstr. 7, \\ Bamberg 96049, Germany \\ e-mail: otoole@sternwarte.uni-erlangen.de \\ Received 22 June 2004 / Accepted 2 July 2004

\begin{abstract}
We report the discovery of strong photospheric resonance lines of Ga III, Ge IV, Sn IV and $\mathrm{Pb}$ IV in the UV spectra of more than two dozen sdB and sdOB stars at temperatures ranging from $22000 \mathrm{~K}$ to $40000 \mathrm{~K}$. Lines of other heavy elements are also detected, however in these cases more atomic data are needed. Based on these discoveries, we present a hypothesis to explain the apparent lack of silicon in sdB stars hotter than $\sim 32000 \mathrm{~K}$. The existence of triply ionised $\mathrm{Ge}, \mathrm{Sn}$, and $\mathrm{Pb}$ suggests that rather than silicon sinking deep into the photosphere, it is removed from the star in a fractionated stellar wind. This hypothesis provides a challenge to diffusion models of sdB stars.
\end{abstract}

Key words. stars: subdwarfs - stars: abundances

\section{Introduction}

Improving atomic data and access to high-resolution UV spectra through HST and FUSE have allowed abundance analyses of hot, degenerate stars to be undertaken (e.g. Ohl et al. 2000; Barstow et al. 2003). We have recently begun a project to measure the abundances of iron-group elements in hot subdwarf B stars (O'Toole et al. 2003; Chayer et al. 2003). These stars can be associated with models of hot, helium core-burning stars with hydrogen layers too thin to sustain nuclear burning. Analyses of high resolution IUE spectra of sdBs revealed peculiar chemical abundances. In particular, carbon varies greatly from star to star, nitrogen is essentially solar, while silicon is slightly underabundant up to $\sim 32000 \mathrm{~K}$, but at hotter temperatures it is very deficient - at least 5 dex below the solar value in some cases (e.g. Lamontagne et al. 1987; Baschek et al. 1982). These chemical pecularities in sdBs are usually explained by invoking a complex balance of radiative leviation and gravitational settling combined with mass loss (Unglaub \& Bues 2001; Bergeron et al. 1988), however, there have only been a few diffusion models published, with varying success (Unglaub \& Bues 2001; Charpinet et al. 1997).

In this letter we present the discovery of strong resonance lines of several heavy elements in $\mathrm{sdB}$ (there will be no distinction made between sdB and sdOB stars here) based on high resolution UV spectra. Three of the elements discovered (germanium, tin and lead) have the same electronic configuration as silicon, and we discuss the implications of this in the context

^ Based on observations made with the NASA/ESA Hubble Space Telescope, which is operated by the Association of Universities for Research in Astronomy, Inc., under NASA contract NAS 5-26555. These observations are associated with program \#8635 and \#5319. of diffusion theory. A detailed abundance analysis is currently being carried out (O'Toole et al., in preparation).

\section{Observations}

Our original observations were made with $H S T / S T I S$ as part of an ongoing project to measure metal abundances in pulsating and non-pulsating sdBs (proposal \#8635, PI: U. Heber). We obtained data for five targets using the E140M grating, which in the far UV has a resolving power of $R=45800$ and a useful wavelength range of $1165-1710 \AA$. Final results will be presented in a future paper, however a preliminary analysis can be found in O'Toole et al. (2003), while the serendipitous discovery of a white dwarf companion to one of the stars (Feige 48) using these data has been presented by O'Toole et al. (2004).

After our original discovery of heavy element resonance lines in our HST/STIS spectra, we searched for any other spectra of $\mathrm{sdB} / \mathrm{O}$ stars in the $H S T$ and IUE archives. This resulted in high-resolution UV HST/STIS spectra of Feige 66 and CPD $-64^{\circ} 481$, a $H S T / G H R S$ spectrum of the sdO Feige 46 , and the IUE spectra of 21 more stars, representing almost the entire range of effective temperatures and surface gravities for the sdB stars (see Fig. 1). In one case (Feige 110) the detection of the $\mathrm{Pb}$ IV line at $1313.072 \AA$ was uncertain, so we looked for the second resonance line at $1028.611 \AA$ using archival FUSE spectra.

\section{Discovery of heavy element resonance lines}

The analysis of the UV spectra of sdBs is complicated by the large number of absorption lines caused by iron-group elements. The wavelengths of these lines in the Kurucz database 


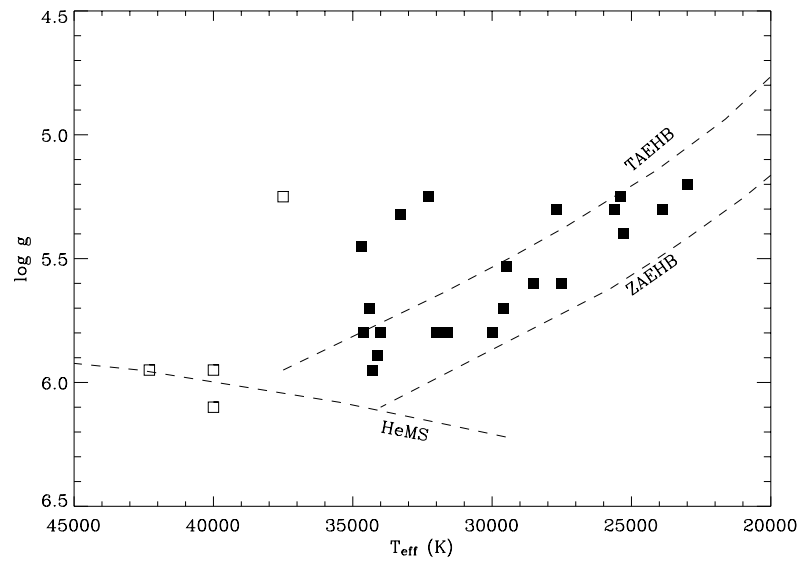

Fig. 1. $T_{\text {eff }}-\log g$ diagram of the sample stars. Filled squares show sdB stars, while open squares show sdOs. Two stars are not shown since they do not have temperature determinations.

in some cases can be quite inaccurate, especially at bluer wavelengths. This can mean that some lines in the spectrum appear to be unidentified. While analysing our HST/STIS spectra we noticed that the wavelengths of two very strong unmatched lines corresponded very well with the resonance doublet of Ge IV. The region around the line at $1189.028 \AA$ is shown in Fig. 2 (the other line is at $1229.840 \AA$ ). There are no strong lines near these resonance lines that could lead to an incorrect identification. Using the line lists of Morton (2003, 2000), we discovered the resonance doublets of Ga III at $1495.045 \AA$ and $1534.462 \AA$ and of Sn IV at 1314.539 $\AA$ and $1437.525 \AA$, as well as one resonance line of Pb IV at $1313.072 \AA$ (the other line is at $1028.611 \AA$, lies in the wings of $\mathrm{Ly} \beta$ ). The strongest Sn III resonance line at $1251.387 \AA$ may also be present, but is blended with an unknown feature. The three resonance lines of $\mathrm{Zr}$ IV, as well as Zn IV and possibly Cd IV are present in at least Feige 66, however no oscillator strengths for these lines could be found in the literature. We note that the only other report of these resonance lines in UV spectra was by Proffitt et al. (2001), who discussed the lead, tin and germanium abundances of the SMC main-sequence B star AV 304. They compared this star with the Galactic B star HD 46328. Both of these stars show the same Ge IV, Sn IV and Pb Iv lines seen in our spectra. It is quite possible that these lines have been previously overlooked in the spectra of other main sequence $O$ and B stars, although with many objects rapidly rotating, their identification might be difficult. This is one clear advantage that hot subdwarfs have: their rotation velocities are typically less than $5 \mathrm{~km} \mathrm{~s}^{-1}$.

The discovery of the resonance lines of these heavy elements encouraged us to look for subordinate lines. These lines should be much weaker, but may still be detectable. We used the NIST Atomic Spectra Database ${ }^{1}$ as well as the line list of Hirata \& Horaguchi (1995). In the case of Ge IV, there are two strong lines around $1494.9 \AA$ and $1500.6 \AA$ that have listed oscillator strengths, however because the wavelengths are not very well known, we cannot claim a certain

${ }^{1}$ See http://physics.nist.gov/cgi-bin/AtData/ lines_form

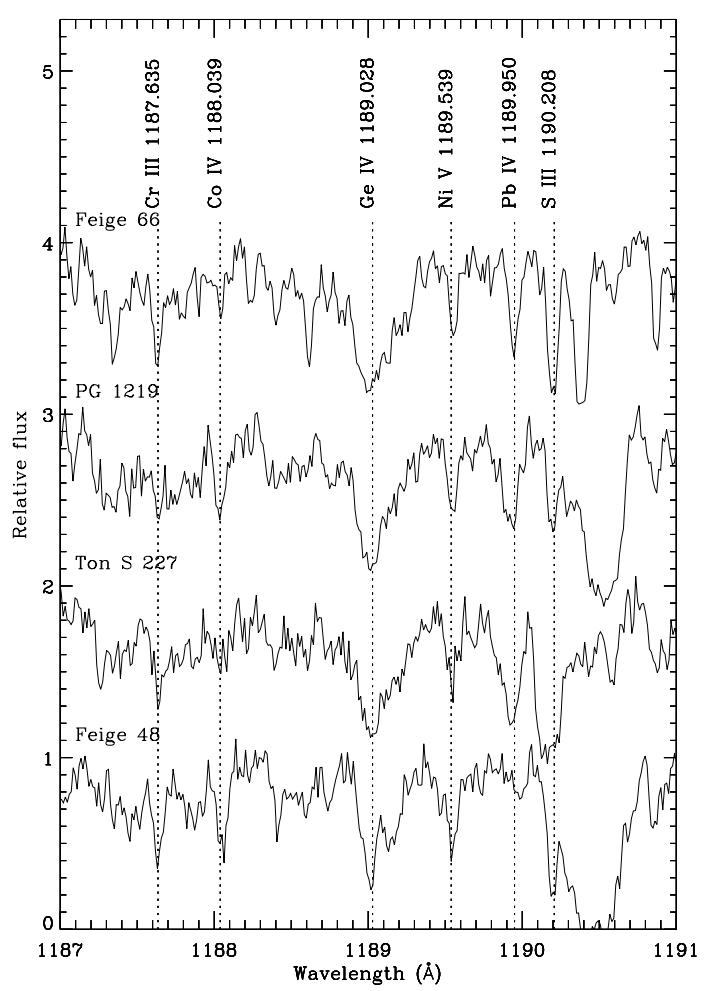

Fig. 2. HST/STIS spectra of $1187-1191 \AA$ region showing the Ge IV $1189.028 \AA$ line, as well as the Pb IV at $1189.95 \AA$. There is no atomic data for the latter. The strong feature around $1190.5 \AA$ is due to interstellar Si II. Several other strong lines are also marked.

detection. There are also a couple of strong Ga III lines with published oscillator strengths, and these are detected at $1267.151 \AA$ and $1293.446 \AA$. Several subordinate $\mathrm{Pb}$ IV lines with reasonably accurate wavelengths are seen, however once again there are no oscillator strengths available in the literature. The strongest appears to be the line at $1189.95 \AA$, which is clearly present in the three hottest stars Feige 66, PG 1219+534 and Ton S 227, but weak in Feige 48 (see Fig. 2). Other weaker $\mathrm{Pb}$ IV are possibly detected at $1343.06 \AA$, $1400.26 \AA$, and $1535.71 \AA$. There are no strong Sn Iv lines other than the resonance lines detectable in our spectra.

Our HST/STIS spectra are not the only high-resolution UV spectra available covering this wavelength range. There were a number of sdBs observed with $I U E$, and their resolution is good enough to be able to detect some of the lines we have discovered here, although in some cases low signal-tonoise makes things difficult. We show a list of all the stars in our sample in Table 1, and a selection of spectra around the $\mathrm{Pb}$ IV and Sn IV resonance lines in Fig. 3. In almost all stars the lines of $\mathrm{Ge}, \mathrm{Sn}$, and $\mathrm{Pb}$ are present. Low signal-to-noise is responsible in most cases for uncertainty, although in some stars the spectra are smeared by rotation (PG 1605+072, SB 290) or tidally-locked binary motion (HW Vir, PG 0113+114). The stars in Table 1 are grouped into sdOs at the top, "cool" sdBs in the middle, and "hot" sdBs (also known as sdOBs) at the bottom. The cool and hot sdBs are divided arbitrarily by the appearance of Si Iv resonance lines (see Sect. 4). HZ 44 is an unusual object, since it is hot but shows the same heavy metal 
Table 1. List of sdB/sdO stars with high-resolution UV spectra, comparing presence of G14 elements. Observations of stars marked with an asterisk were made with $H S T / S T I S$; all other observations were made with IUE. The spectra of HW Vir and PG 0133+114 are smeared by binary motion, while the PG $1605+072$ and SB 290 spectra are smeared by rotation.

\begin{tabular}{lccccc}
\hline \hline Star & Si & Ge & Sn & Pb & Binary \\
\hline HZ 44 & $\mathrm{Y}$ & $\mathrm{Y}$ & $\mathrm{Y}$ & $\mathrm{Y}$ & $?$ \\
SB 884 & $\mathrm{Y}$ & $\mathrm{Y}$ & $?$ & $\mathrm{Y}$ & $?$ \\
Feige $46^{*}$ & $\mathrm{Y}$ & $?$ & $?$ & $\mathrm{Y}$ & $?$ \\
Feige 110 & $\mathrm{~N}$ & $\mathrm{Y}$ & $?$ & $?$ & $?$ \\
SB 290 & $\mathrm{Y}$ & $?$ & $?$ & $?$ & $?$ \\
HD 4539 & $\mathrm{Y}$ & $\mathrm{Y}$ & $\mathrm{Y}$ & $\mathrm{Y}$ & $\mathrm{N}$ \\
HD 171858 & $\mathrm{Y}$ & $\mathrm{Y}$ & $\mathrm{Y}$ & $\mathrm{Y}$ & $\mathrm{Y}$ \\
HD 185510 & $\mathrm{Y}$ & $?$ & $\mathrm{Y}$ & $\mathrm{Y}$ & $\mathrm{Y}$ \\
HD 205805 & $\mathrm{Y}$ & $\mathrm{Y}$ & $\mathrm{Y}$ & $\mathrm{Y}$ & $\mathrm{N}$ \\
FF Aqr & $\mathrm{Y}$ & $\mathrm{Y}$ & $\mathrm{Y}$ & $\mathrm{Y}$ & $\mathrm{Y}$ \\
HW Vir & $\mathrm{Y}$ & $?$ & $?$ & $?$ & $\mathrm{Y}$ \\
Feige 48* & $\mathrm{Y}$ & $\mathrm{Y}$ & $\mathrm{Y}$ & $\mathrm{Y}$ & $\mathrm{Y}$ \\
Feige 65 & $\mathrm{Y}$ & $?$ & $\mathrm{Y}$ & $\mathrm{Y}$ & $\mathrm{N}$ \\
CPD-64 481* & $\mathrm{Y}$ & $\mathrm{Y}$ & $\mathrm{Y}$ & $\mathrm{Y}$ & $?$ \\
PG 0133+114 & $\mathrm{Y}$ & $?$ & $?$ & $?$ & $\mathrm{Y}$ \\
PG 0342+026 & $\mathrm{Y}$ & $\mathrm{Y}$ & $\mathrm{Y}$ & $\mathrm{Y}$ & $\mathrm{N}$ \\
PG 1605+072* & $\mathrm{Y}$ & $\mathrm{Y}$ & $?$ & $?$ & $?$ \\
PG 1032+406 & $\mathrm{N}$ & $\mathrm{Y}$ & $\mathrm{Y}$ & $\mathrm{Y}$ & $\mathrm{Y}$ \\
PG 1104+243 & $\mathrm{N}$ & $\mathrm{Y}$ & $\mathrm{Y}$ & $\mathrm{Y}$ & $\mathrm{Y}$ \\
PG 1219+534* & $\mathrm{N}$ & $\mathrm{Y}$ & $\mathrm{Y}$ & $\mathrm{Y}$ & $?$ \\
PG 1352-022 & $\mathrm{N}$ & $\mathrm{Y}$ & $?$ & $\mathrm{Y}$ & $?$ \\
HD 149382 & $\mathrm{N}$ & $\mathrm{Y}$ & $\mathrm{Y}$ & $\mathrm{Y}$ & $\mathrm{N}$ \\
Feige 66* & $\mathrm{N}$ & $\mathrm{Y}$ & $\mathrm{Y}$ & $\mathrm{Y}$ & $\mathrm{N}$ \\
Ton S 227* & $\mathrm{N}$ & $\mathrm{Y}$ & $\mathrm{Y}$ & $\mathrm{Y}$ & $?$ \\
BD+34 1543 & $\mathrm{~N}$ & $\mathrm{Y}$ & $?$ & $\mathrm{Y}$ & $\mathrm{Y}$ \\
PHL 932 & $\mathrm{N}$ & $\mathrm{Y}$ & $\mathrm{Y}$ & $\mathrm{Y}$ & $\mathrm{N}$ \\
UVO 1758+36 & $\mathrm{N}$ & $\mathrm{Y}$ & $\mathrm{Y}$ & $\mathrm{Y}$ & $\mathrm{N}$ \\
\hline & & & & & \\
\hline
\end{tabular}

lines as cooler sdB stars. This may be due to convection related to the stars high helium abundance $(\log (\mathrm{He} / \mathrm{H}) \sim 0.4)$. The sdO stars SB 884 and Feige 46 are peculiar and may not have evolved from the Horizontal Branch. The IUE spectrum of the hydrogen-rich sdO Feige 110 has several bad pixels in the $1310-1315 \AA$, making identification of lines in this region difficult. We note that the second $\mathrm{Pb}$ IV resonance line at $1028.611 \AA$ may be present in the FUSE spectrum of Feige 110, however it appears to be blended, meaning that the presence of lead remains uncertain. The case of the Sn Iv $1437.525 \AA$ line is similar. The last column in Table 1 shows the binary status of the stars; the presence of heavy element lines appears to be independent of binarity. Finally, three of the stars in our sample are pulsating sdBs, and two of them clearly show the lines discussed, while the third (PG 1605+072) is rapidly rotating, making line identification difficult.

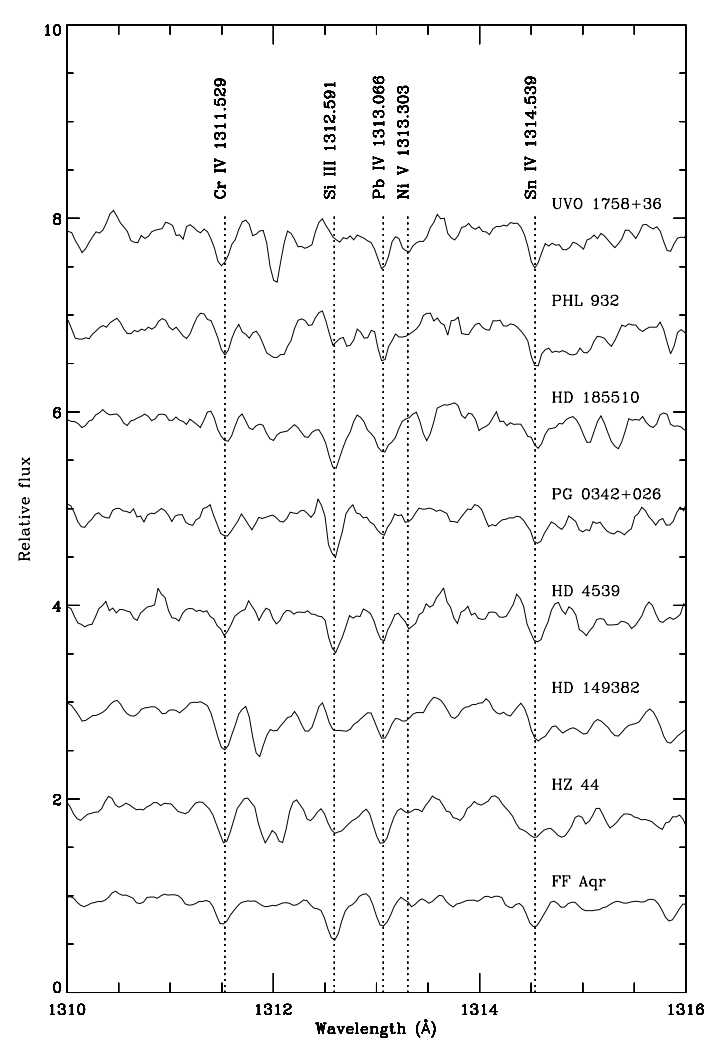

Fig. 3. IUE spectra of the 1311-1315 $\AA$ region showing the Sn IV and $\mathrm{Pb}$ Iv resonance lines. The spectra have been offset for clarity and smoothed with a boxcar filter two pixels wide.

\section{Implications}

The effects of diffusion hamper any meaningful discussion about the origin of the heavy elements discussed in this letter (i.e. whether they are formed by the $r$ - or $s$-process). Clearly they can not be formed in these hot subdwarfs, but their presence can, however, be used to shed some light on some of the other chemical peculiarities found in these stars.

It has long been know that silicon in sdB stars show peculiar behaviour. Above a certain temperature $(\sim 32000 \mathrm{~K})$ there is almost no silicon left in the upper photosphere. Previous studies suggested that above this temperature silicon might exist in a noble gas configuration (e.g. Baschek et al. 1982), and that it should sink deep into the photosphere since these atoms would absorb little radiative flux, and hence momentum. Calculations by Michaud et al. (1985) and Bergeron et al. (1988) have found, however, that diffusion alone cannot account for the large silicon underabundances, and that a competing particle transport mechanism, i.e. a weak stellar wind, must be present. The discovery of strong $\mathrm{Ge}, \mathrm{Sn}$ and $\mathrm{Pb}$ resonance lines can be used to confirm this. Consider that $\mathrm{Si}, \mathrm{Ge}, \mathrm{Sn}$ and $\mathrm{Pb}$ occupy the same column in the periodic table (Group 14, hereafter G14), i.e. they each have 4 electrons in their outer shell. (Note that C Iv, the lowest mass G14 element, has a significantly higher ionisation potential $(64.492 \mathrm{eV})$, and is also produced by the triple- $\alpha$ process, meaning that it is somewhat independent of the other G14 elements.) This means that we can expect their susceptibility to absorb radiative momentum to be quite similar. In other words, to a first approximation, 
the elements' initial abundances and relative masses will be the only things that affect the outcome of their acceleration by radiative flux. As a consequence, the velocity of $\mathrm{Pb}$ atoms will be seven times lower than $\mathrm{Si}$ atoms, since $\mathrm{Si}$ is seven times lighter than lead. Consider that the escape velocity of a typical sdB is $\sim 1200 \mathrm{~km} \mathrm{~s}^{-1}$; if $v(\mathrm{Si})=2000 \mathrm{~km} \mathrm{~s}^{-1}$ (not an unrealistic value), then $v(\mathrm{~Pb})=\sim 270 \mathrm{~km} \mathrm{~s}^{-1}, v(\mathrm{Sn})=\sim 475 \mathrm{~km} \mathrm{~s}^{-1}$, and $v(\mathrm{Ge})=\sim 770 \mathrm{~km} \mathrm{~s}^{-1}$. In other words, silicon will escape the star, but the heavier elements will not. A similar qualitative argument was put forward by Leckrone et al. (1999) to explain the abundance peculiarities of the HgMn star $\chi$ Lupi.

An implication of the hypothesis presented here is that this wind must be fractionated, rather than uniform, since Coulomb coupling is inefficient in extremely thin winds. Fontaine \& Chayer (1997) investigated the effects of a weak stellar wind on helium in sdBs and found that the observed abundances can be explained if the mass-loss rates are between about $10^{-14}$ and $10^{-13} M_{\odot} \mathrm{yr}^{-1}$. Unglaub \& Bues (2001) found that the CNO elements could show strong deficiencies as well as enrichments when they considered the effects of such a wind with these kinds of mass-loss rates. Detecting such a wind directly would be difficult for most sdBs, but Heber et al. (2003) discovered four luminous objects that show weak $\mathrm{H} \alpha$ emission cores, and suggested that these may be the signature of a stellar wind. Preliminary calculations by Vink (2004) show that the massloss rates for these stars may be as high as $10^{-11} M_{\odot}$. Because none of sdB stellar wind studies mentioned here have done so, we suggest that future calculations include silicon.

Naively one might expect atoms with similar mass to be affected in the same way as silicon. For lighter elements this appears to be the case, with aluminium also at least significantly depleted in hotter sdBs (e.g. Baschek et al. 1982). The presence of Ga III, with a similar ionisation potential, suggests that $\mathrm{Al}$ III should be present in these stars, rather than Al IV. For the main ionisation stages of the other elements ( $\mathrm{Mg}$ III, P III/IV, S III/IV, Ar III) in Period 3, however, most flux is absorbed by lines below the Lyman limit, where very little is emitted by sdB stars. As a consequence, these elements will not be as strongly driven as aluminium or silicon, meaning they should be present in the spectra of sdB stars. This is the case for all species except $\mathrm{Mg}$ III, which has very few strong lines at observable wavelengths.

\section{Summary}

We have discovered strong resonance lines of lead, tin, germanium and gallium in many sdB stars. The presence of these lines is independent of binarity, and they are present in both pulsating and non-pulsating sdBs. Other heavy elements may also be present, however lists of laboratory wavelengths and oscillator strengths are missing or incomplete. The lines should also be present main sequence $\mathrm{O}$ and $\mathrm{B}$ stars. We suggest that the presence of heavy Group 14 elements, but the near absence of silicon in the hotter sdBs, implies that rather than sinking deeping into the photosphere, silicon may have been carried out of the star by a fractionated stellar wind. This hypothesis is supported by measurements of aluminium resonance lines, which also show strong depletion in hotter sdB stars. These discoveries provide a challenge for future diffusion and stellar wind models of hot subdwarfs.

Acknowledgements. We are thankful to Norbert Przybilla for many helpful comments, as well as Uli Heber and Klaus Unglaub for useful discussions. S.J.O.T. is supported by the Deutsches Zentrum für Luftund Raumfahrt (DLR) through grant No. 50-OR-0202.

\section{References}

Barstow, M. A., Good, S. A., Holberg, J. B., et al. 2003, MNRAS, 341,870

Baschek, B., Hoeflich, P., \& Scholz, M. 1982, A\&A, 112, 76

Bergeron, P., Wesemael, F., Michaud, G., \& Fontaine, G. 1988, ApJ, 332, 964

Charpinet, S., Fontaine, G., Brassard, P., et al. 1997, ApJ, 483, L123

Chayer, P., Fontaine, G., Fontaine, M., et al. 2003, in Extreme Horizontal Branch stars and related objects, ed. P. Maxted (Kluwer), in press

Fontaine, G., \& Chayer, P. 1997, in The third Conf. on faint blue stars, 169

Heber, U., Maxted, P. F. L., Marsh, T. R., Knigge, C., \& Drew, J. E. 2003, in NATO ASIB Proc. 105: White dwarfs, 109

Hirata, R., \& Horaguchi, T. 1995, VizieR on-line data catalog: VI/69 Lamontagne, R., Wesemael, F., \& Fontaine, G. 1987, ApJ, 318, 844

Leckrone, D. S., Proffitt, C. R., Wahlgren, G. M., Johansson, S. G., \& Brage, T. 1999, AJ, 117, 1454

Michaud, G., Bergeron, P., Wesemael, F., \& Fontaine, G. 1985, ApJ, 299, 741

Morton, D. C. 2000, ApJS, 130, 403

Morton, D. C. 2003, ApJS, 149, 205

Ohl, R. G., Chayer, P., \& Moos, H. W. 2000, ApJ, 538, L95

O'Toole, S. J., Heber, U., \& Benjamin, R. A. 2004, A\&A, in press

O'Toole, S. J., Heber, U., Chayer, P., et al. 2003, in Extreme horizontal branch stars and related objects, ed. P. Maxted (Kluwer) [arXiv: astro-ph/0309062]

Proffitt, C. R., Sansonetti, C. J., \& Reader, J. 2001, ApJ, 557, 320

Unglaub, K., \& Bues, I. 2001, A\&A, 374, 570

Vink, J. 2004, in Extreme Horizontal Branch stars and related objects, ed. P. Maxted (Kluwer) [astro-ph/0309011] 